\title{
Recirculating Apparatus for Testing Hygrometers ${ }^{1}$
}

\author{
By Arnold Wexler
}

\begin{abstract}
An apparatus that was developed for producing atmospheres of known relative humidity is described. It was designed, principally, for research on and calibration of radiosonde hygrometer elements at temperatures above freezing, but is equally useful at temperatures down to $-40^{\circ} \mathrm{C}$. It operates by saturating air at one temperature and then raising the air temperature sufficiently to give any desired relative humidity. The air is recirculated continuously in a closed system. Four identical, but independent, recirculating systems are employed. Discrete changes in humidity and temperature are obtained by means of a novel pneumatic valve that permits the hygrometers under test to be switched, easily, from system to system. Checks on the performance of the apparatus by means of the gravimetric method of moisture determination and the psychrometric method show an average difference in relative humidity of \pm 1.2 percent.
\end{abstract}

\section{Introduction}

In the meteorological sounding of the upper atmosphere by means of the radiosonde, the device commonly employed for sensing the water vapor content of the air is the electric hygrometer. ${ }^{2}{ }^{3}$ During a flight, the hygrometer may be subjected to a wide range of temperatures and humidities, as well as to air flows, corresponding to rates of ascent of the balloon, of $1,000 \mathrm{ft} / \mathrm{min}$ or more. These conditions of use have created a need for some convenient means of producing and maintaining atmospheres of known relative humidity, at any of the temperatures encountered in a radiosonde flight, for calibration and research purposes.

An apparatus has been developed to fulfill this need. It was designed for operation, principally, at temperatures above $0^{\circ} \mathrm{C}$ but is equally useful at temperatures down to $-40^{\circ} \mathrm{C}$. Provision was made for obtaining discrete changes in humidity and temperature and for maintaining given air speeds. It supplements an apparatus of the divided-flow type previously built for use at temperatures below freezing. ${ }^{4}$

\section{Theory}

A fundamental method for producing an atmosphere of known relative humidity is to saturate a stream of air with water vapor at a given temperature and then to raise the temperature of the air to a specified higher value. If $t_{s}$ is the temperature of saturation, $e_{s}$ the saturation pressure of water vapor at temperature $t_{s}, t_{t}$ the elevated temperature, and $e_{t}$ the saturation pressure of water vapor at temperature $t_{t}$, then the relative humidity, $H$, at temperature $t_{b}$ is

$$
H=100 \frac{e_{s}}{e_{t}} \text {. }
$$

In eq 1 the assumption is made that the absolute pressure of the air at the elevated temperature, $t_{t}$, is the same as the absolute pressure of the air at the saturation temperature, $t_{s}$. If these two pressures

${ }_{1}^{1}$ This apparatus was developed for the Bureau of Ships, Department of the Navy.

2 F. W. Dunmore, J. Research NBS 20, 723 (1938) RP1102.

${ }^{3}$ F. W. Dunmore, J. Research NBS 23, 701 (1939) RP1265.

4 A. Wexler, J. Research 40, 479 (1948) RP1894. are not identical, then a pressure correction must be introduced. If $P_{s}$ is the absolute pressure of the air at $t_{s}$, and $P_{t}$ is the absolute pressure of the air at $t_{t}$, then the relative humidity is

$$
H=100 \frac{e_{s}}{e_{t}} \frac{P_{t}}{P_{s}}
$$

A simplified schematic diagram illustrating how the two-temperature method may be employed in an apparatus is shown in figure 1. By means of a gas pump, air is circulated from a saturator into a test chamber and then back into the saturator. The temperature, $t_{s}$, of the saturator and the temperature, $t_{t}$, of the test chamber are maintained at their respective desired values by thermostatted baths. Complete saturation is achieved simply, and efficiently by recirculation of air over water in the saturator, in a closed system. The relative humidity, $H$, in the test chamber is given by eq 2 , where the saturation vapor pressures, $e_{s}$ and $e_{t}$, corresponding to temperatures $t_{s}$ and $t_{t}$, are obtained from standard tables, and the absolute pressures, $P_{s}$ and $P_{t}$, are measured values.

The design of a practical system, based on this method, is shown in figure 2. It differs from the elementary design of figure 1 in that the testchamber temperature is controlled by means of a heater inserted in the air stream rather than by

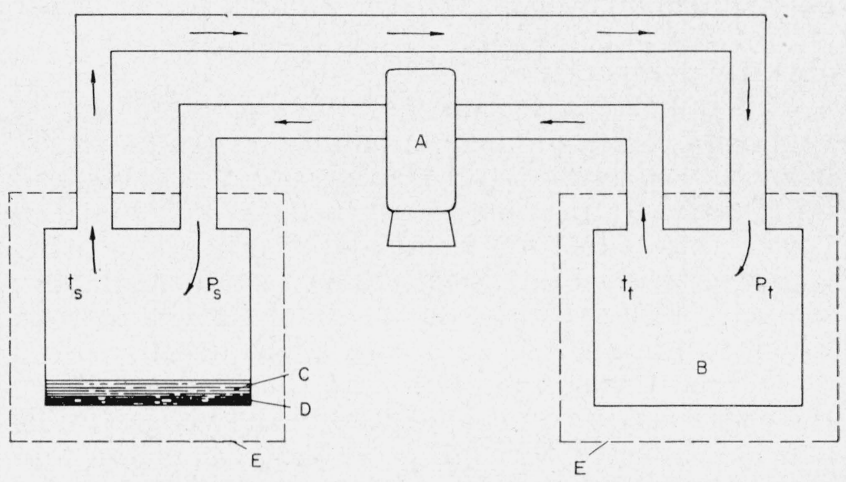

Figure 1. Simplified schematic diagram illustrating the twotemperature recirculating method of relative humidity production.

A, Gas pump; B, test chamber; C, water; D, saturator; E, thermostatted bath. 


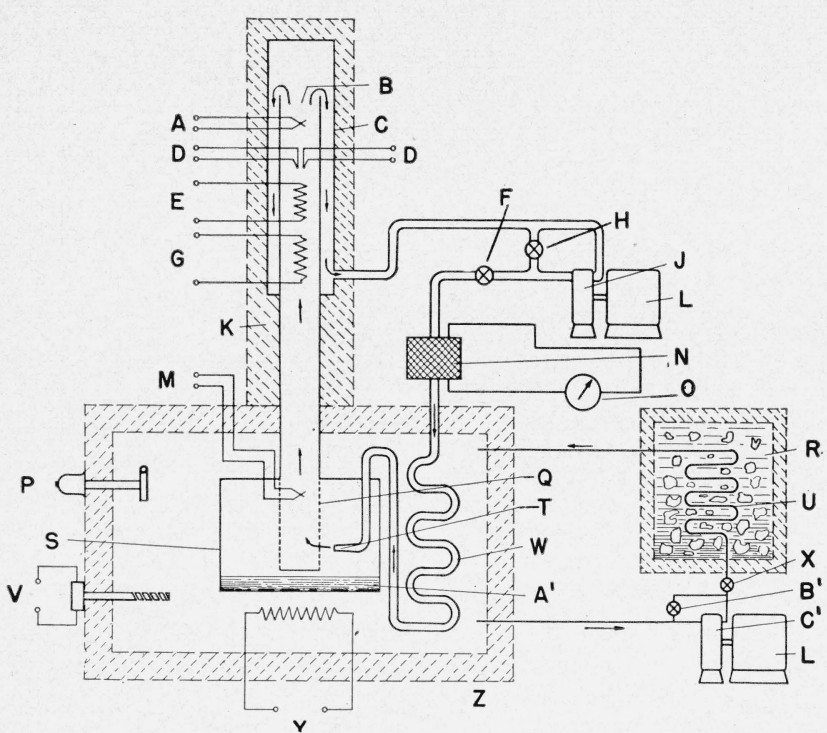

Figure 2. Schematic diagram of a practical two-temperature recirculating humidity apparatus.

A, Test-chamber thermocouple; B, test chamber; C, reflux tube; D, thermistor; $\mathrm{E}$, intermittent heater; $\mathrm{F}$, control valve (air); G, gross heater; $\mathrm{H}$, bypass valve (air); J, gas pump; K, thermal insulation; L, motor; M, saturator thermocouple $\mathrm{N}$, flow meter; O, differential pressure gage; P, centrifugal stirrer; Q, screen; $\mathrm{R}$, dry-ice bath; S, saturator; T, nozzle, U, coil (liquid); V, thermoregulator; R, dry-ice bath; S, saturator; T, nozzle, U, coil (liquid); V, thermoregulator;
W, coil (air); X, control valve (liquid); Y, bath heater; $\mathrm{Z}$, thermostatted Varsol bath; A', water; $\mathrm{B}^{\prime}$, bypass valve (liquid); $\mathrm{C}^{\prime}$, circulating pump.

immersing the test chamber in a thermostatted liquid bath. The elimination of a thermostatted bath for the test chamber, with the resultant simplification in design and construction, is made possible by the fact that the saturator must necessarily be maintained at a lower temperature than the test chamber, and hence the only requirement for achieving any desired test-chamber temperature is the introduction of the requisite amount of heat into the air stream. A novel feature of the test-chamber design is the concentric tube arrangement. The air from the saturator flows upward through the inner tube, then reverses its direction and returns downward in the annular space between the inner and outer tubes. The outer tube is thermally insulated. This reflux action contributes to temperature control and a reduction in thermal losses, for it surrounds the test space, which is at the upper end of the inner tube, with air at or near the testchamber temperature.

The important features of the saturator are its simplicity of design, its adaptability for temperatures below as well as above the freezing point of water, and its effective saturation of air at both high and low flows. These features are achieved in large measure by imparting a centrifugal action to the entrant air stream. The saturator is a cylindrical chamber to which water is added to a convenient depth. Air is discharged through a nozzle into the chamber above the water surface and tangential to the vertical walls and is exhausted through a central port in the top. The centrifugal action creates a whirlpool and thoroughly mixes water vapor with the air. Spray and liquid droplets are forced to the walls by centrifugal force, with the result that there is little tendency for liquid water to emerge through the exit port, except at very low air flows. A multilayer fine wire screen baffle is used at the exit to trap and prevent water from passing out of the saturator at the low flows. As the air does not bubble through the water but only passes over its exposed surface, the water may be frozen without impairing the functioning of the saturator. The saturation pressure of the water vapor in the saturator (for use in eq 2) is taken, then, with respect to ice.

A desirable requirement in a humidity apparatus is a scheme or mechanism whereby the relative humidity may be changed quickly and in discrete steps. Such a scheme is necessary for lag studies on hygrometers. It is achieved in this equipment by utilizing four identical but independent systems that are arranged in such a fashion that their test chambers may be interchanged easily and rapidly. Thus, if a different temperature, $t_{s}$, is maintained in each of the four saturators, and if the same temperature, $t_{t}$, is maintained in each of the four test chambers, then the relative humidity in each test chamber is different. By interchanging the test chambers, the relative humidities therein undergo discrete changes, as each test chamber now communicates with a different saturator.

The arrangement is shown schematically in figure 3 for two of the four systems. The interchange of test chambers is effected by a special pneumatic switch. It consists of two ground and lapped plates to which the inner and outer tubes are attached and which contain ports through which the air may pass. A quarter turn of the top plate with respect to bottom plate advances each test chamber to a new position and connects each test chamber with a different

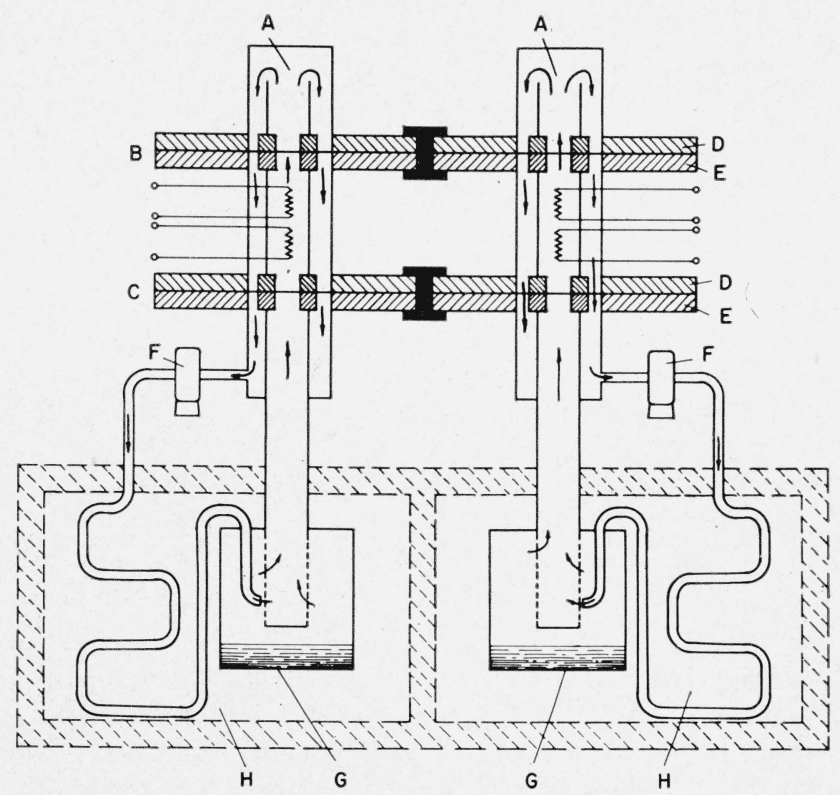

FIGURE 3. Schematic diagram of pneumatic switches for interchanging test chambers; only two of the four systems are shown.

A, Test chamber; B, upper switch; C, lower switch; D, top plate; E, bottom plate; $F$, gas pump; $G$, saturator; $H$, thermostatted bath. 
saturator. Two such switches are used instead of one for greater convenience and versatility. For lag studies only, the upper switch is used. The speed with which the relative humidity can be changed is of the order of 1 to 2 seconds and depends primarily on the speed with which the switch can be rotated.

\section{Design Details}

An oil-free impeller-type gas pump is used to circulate air continuously through each system. The flow is controlled by means of a bypass valve, as shown in figure 2, and is measured by a glass-wool linear flowmeter that is calibrated in terms of air speed through the test chamber. The air flows through a copper tube coil, immersed in the same thermostatted liquid bath as the saturator and reaches bath temperature before it passes into the saturator. The cooling coil is essential for satisfactory operation, for unless the air enters the saturator at the bath temperature, an uncertainty in saturation temperature ensues. The saturator is a brass cylinder, 5 in. in diameter and 4 in. deep. The inner tube of the reflux portion of the system is polystryrene with 1/4-in. outside diameter and 1/8-in. wall thickness, while the outer is brass tubing with 2 -in. outside diameter and $1 / 32$-in. wall thickness.

The copper tube coil and saturator are immersed in a varsol bath, whose temperature is closely regulated. Varsol is used so that low temperatures may be attained. A bimetal thermoregulator controls a heater through a power relay. The circuit is shown in figure 4. A positive rotary displacement pump

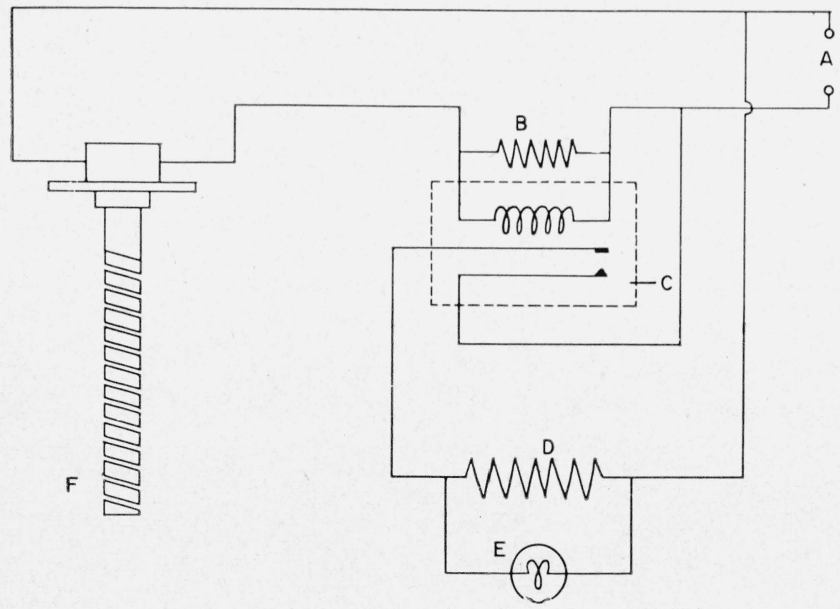

FIguRE 4. Varsol bath temperature control circuit. A, 110-v alternating current; B, shunt resistor; C, power relay; D, bath heater; E, pilot lamp; F, bimetallic thermostat.

withdraws varsol from the bath, circulates it through another cooling coil and discharges it back into the bath. This second cooling coil is maintained at $-78^{\circ} \mathrm{C}$ by crushed dry ice. The cold varsol flow is adjusted, by means of valves, so that the bath temperature will tend to drop very slightly. The heater is arranged to go on when the bath temperature drops below the control point and will go off when the bath temperature is at or above the control point. On-off regulation is thereby achieved. The bath is actively agitated by a centrifugal stirrer.

The temperature of the moving air stream in the test chamber is controlled by two heaters; a gross heater supplies constant heat, the amount manually adjusted, by means of a rheostat, as shown in figure 5; an intermittent heater supplies heat on demand, the amount also manually adjusted by a rheostat and the demand determined by two thermistors acting as the temperature sensitive elements. The gross heater supplies most of the heat, whereas the intermittent heater supplies heat, as required, to raise the temperature to the control point. Figure 6

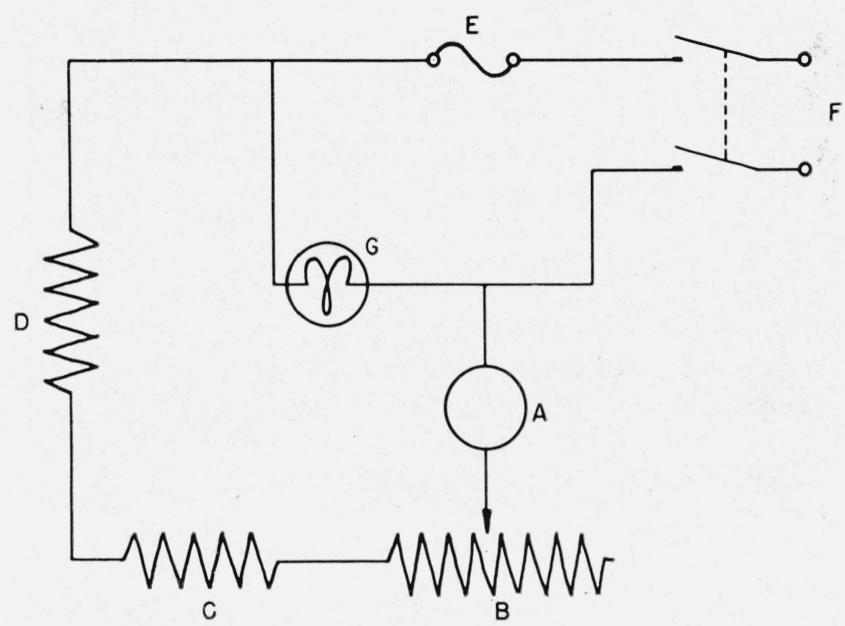

FiguRE 5. Test-chamber gross-heater circuit.

A, Ammeter; B, rheostat; C, limiting resistor; D, gross heater; E, fuse; F, 110-v alternating-current regulated supply; G, pilot lamp

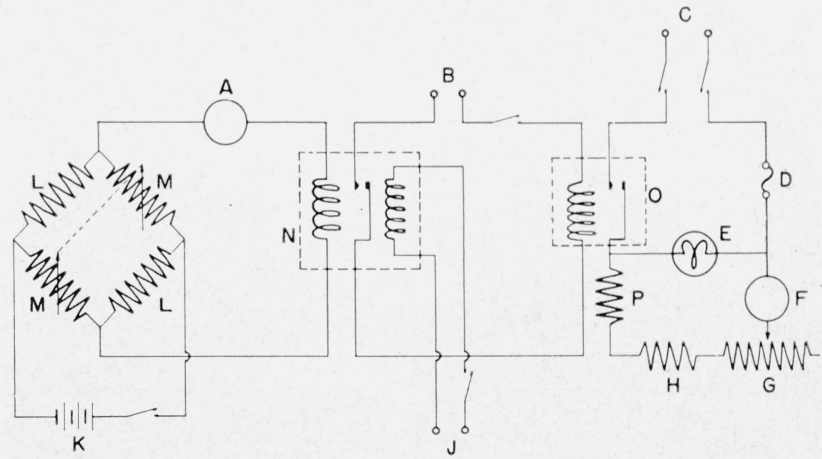

FiguRE 6. Test-chamber intermittent-heater control circuit. A, Microammeter; B, 6-v direct current; C, regulated 110-v alternating current; $\mathrm{D}$, fuse; $\mathrm{E}$, light; $\mathrm{F}$, milliammeter; $\mathrm{G}$, rheostat; $\mathrm{H}$, limiting resistor; J, 28-v pulsing

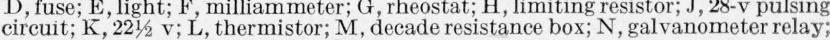
circuit; $\mathrm{K}, 221 / 2 \mathrm{~V} ; \mathrm{L}$, thermistor; $\mathrm{M}$, dec
$\mathrm{O}$, power relay; $\mathrm{P}$, intermittent heater.

is a circuit diagram of the intermittent heater control circuit. The desired control temperature is established by resistances, $M$ (coupled decade resistance boxes) in a Wheatstone bridge circuit containing thermistors, $L$. The bridge balance is sensed by a galvanometer relay. A current of $1 / 2$ microampere deflects the pointer against a magnetic contact, actuating a power relay and, in turn, the intermittent heater. The operation of an electromagnetic 
plunger returns the pointer to a sensing position. If the bridge is balanced, the pointer does not move; if the bridge is unbalanced, the pointer deflects. An electronic pulsing circuit periodically triggers the plunger at a frequency that is adjustable from 2 times a minute to 60 times a minute, so that the galvanometer may sense the balance of the bridge.

The completed apparatus is shown in figure 7 . Figure 8 shows the apparatus with the cover raised showing the four thermostatted baths.

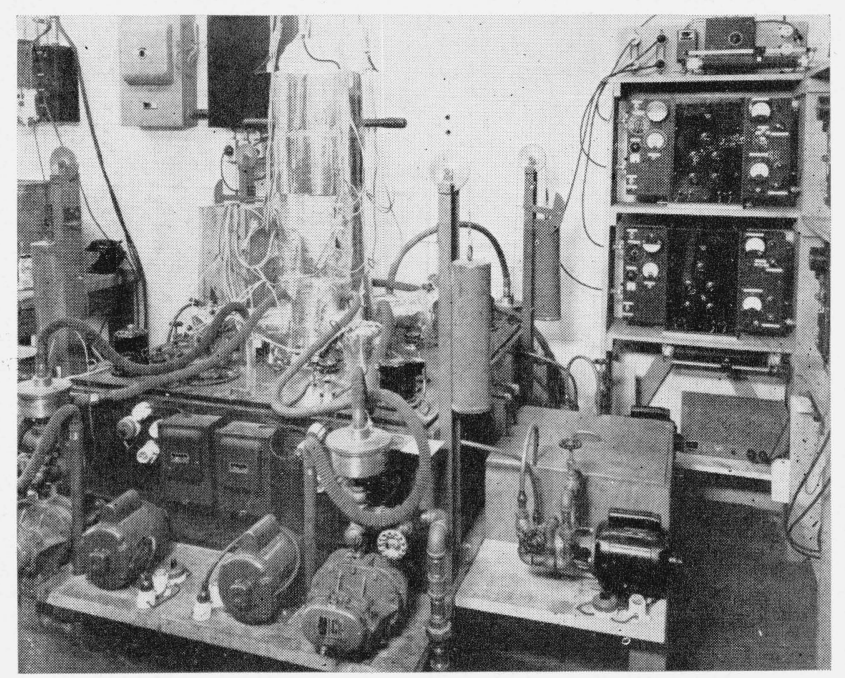

FIgURE 7. Humidity apparatus.

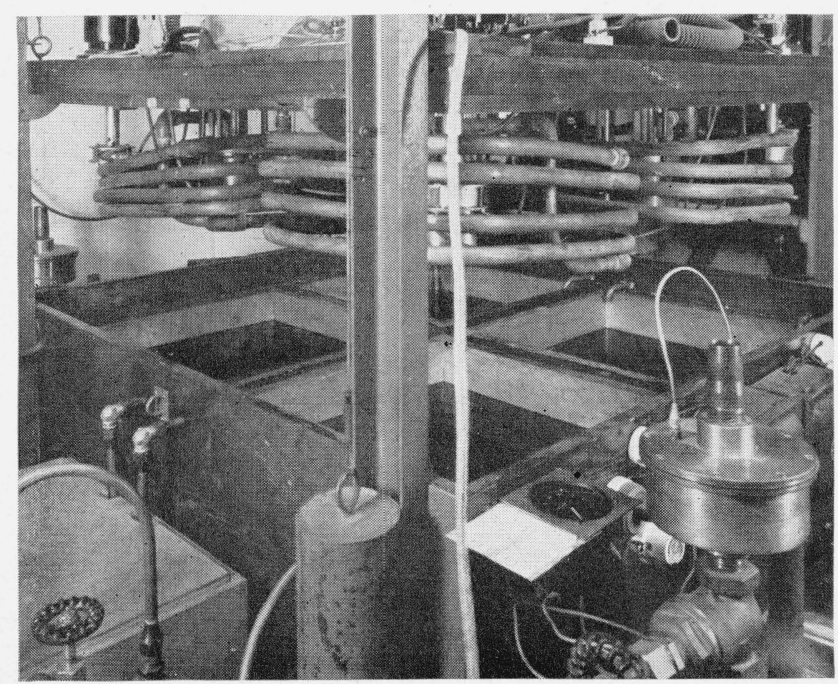

FIGURE 8. View of apparatus with cover raised showing the four independent thermostatted baths.

\section{Performance Characteristics}

The performance of the apparatus has been investigated to determine the accuracy and the usable temperature and humidity ranges. The gravimetric method of moisture determination (see footnote 4) was used to give an independent check on the relative humidity produced by the apparatus for various saturator and test-chamber temperatures and vari- ous speeds of air flow. The results are given in table 1. As an additional check on the accuracy, a thermocouple psychrometer was employed to measure the actual relative humidity in the test chamber. The results are given in table 2 . These tests show an average difference in percentage of relative humidity of \pm 1.2 percent between the gravimetrically measured relative humidity and the apparatus relative humidity given by eq 2 ; and an average difference in percentage of relative humidity of \pm 0.9 percent between the psychrometric measurements and the apparatus values given by eq 2 .

TABLE 1. Gravimetric calibration

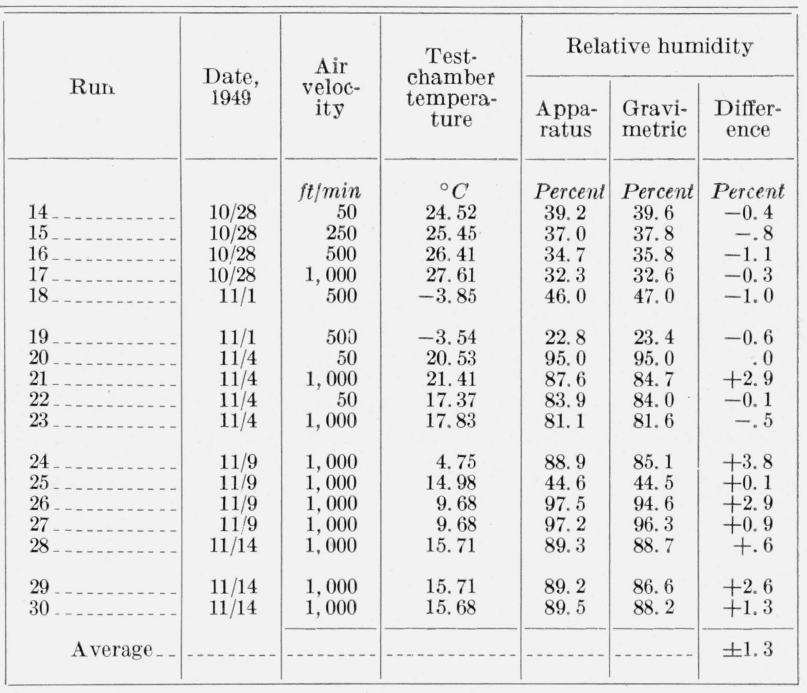

Table 2. Psychrometric calibration

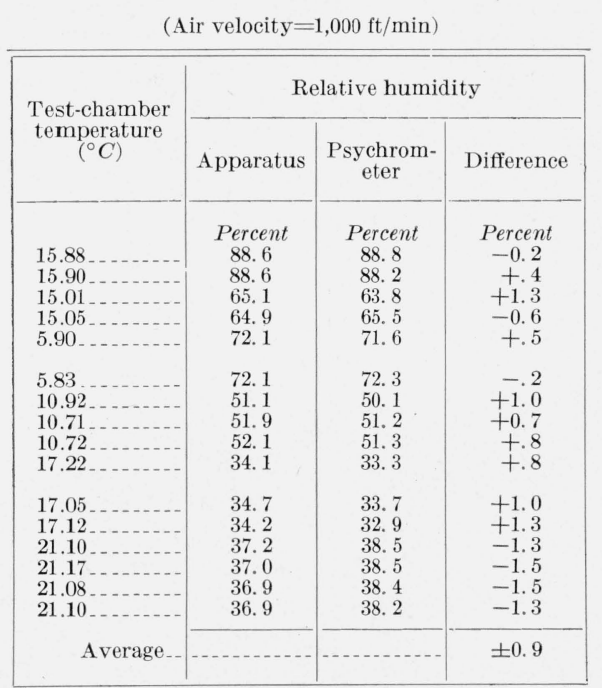

As the precision and accuracy of the apparatus is to a large extent a function of the precision and accuracy of the temperature regulation and measurement of the test chambers and saturators, these temperatures must be adequately controlled and suitably measured. Temperatures are measured 
with calibrated copper-constantan thermocouples and a precision potentiometer to a precision of $0.01 \mathrm{deg}$ C. Because of the fair size of the liquid bath, bimetallic regulation is adequate for maintaining saturation temperatures constant to \pm 0.02 $\operatorname{deg} \mathrm{C}$ over time intervals of 1 hour and constant to $\pm 0.1 \mathrm{deg} \mathrm{C}$ over long time intervals. The thermistor-Wheatstone bridge circuit maintains test chamber temperatures constant to $\pm 0.04 \mathrm{deg} C$ over time intervals of 1 hour and constant to \pm 0.1 $\operatorname{deg} \mathrm{C}$ for long time intervals.

The distribution of temperature within the saturator was explored by means of thermocouples located at the inlet, in the water, and at the outlet. The temperature of the air emerging from the saturator is within $\pm 0.04 \mathrm{deg} \mathrm{C}$ of the temperature of the air entering the saturator; the temperature of the air entering the saturator is within $\pm 0.06 \mathrm{deg}$ $\mathrm{C}$ of the temperature of the water in the saturator; and the temperature of the air emerging from the saturator is within $\pm 0.08 \mathrm{deg} \mathrm{C}$ of the temperature of the water. These temperature differentials may account for errors of $\pm 1 / 2$-percent relative humidity.

The difference in temperature between the saturator and bath was also investigated. The air entering the saturator is within $\pm 0.07 \mathrm{deg} \mathrm{C}$ of the bath temperature; the water temperature is within $\pm 0.04 \mathrm{deg} C$ of the bath temperature; and the air emerging from the saturator is within $\pm 0.09 \mathrm{deg} C$ of the bath temperature.

Although the gas pump is capable of producing very high rates of flow, the coupling between motor and pump is set to allow a maximum air speed of $1,500 \mathrm{ft} / \mathrm{min}$ in the test chamber. By proper adjustment of the bypass valve, any lesser air speed is obtainable.

The upper relative humidity limit is determined by the saturation temperature, the air velocity and the efficiency of the thermal insulation and refluxing action surrounding the test chamber. The thermal leakage through the insulation is $2.2 \mathrm{cal} / \mathrm{min}$ for each degree centigrade temperature difference between the test chamber and room. The maximum relative humidities that may be obtained with air speeds of 50 and $1,000 \mathrm{ft} / \mathrm{min}$ for various saturator temperatures and with the apparatus in a room at $30^{\circ} \mathrm{C}$ are given in table 3 . It is apparent that at high air velocities, the thermal leakage does not seriously limit the relative humidity range. At very low air flows, the maximum relative humidity ob-

TABLE 3. Computed maximum attainable relative humidities

\begin{tabular}{|c|c|c|}
\hline \multirow{2}{*}{$\begin{array}{l}\text { Saturator } \\
\text { temperature } \\
\left({ }^{\circ} \mathrm{C}\right)\end{array}$} & \multicolumn{2}{|c|}{$\begin{array}{l}\text { Maximum relative humid- } \\
\text { ity at test-chamber air } \\
\text { velocity of- }\end{array}$} \\
\hline & $50 \mathrm{ft} / \mathrm{min}$. & $1,000 \mathrm{ft} / \mathrm{min}$ \\
\hline $\begin{array}{r}25 \\
15 \\
5 \\
-5 \\
-20\end{array}$ & $\begin{array}{c}\text { Percent } \\
95 \\
86 \\
77 \\
64 \\
49\end{array}$ & $\begin{array}{c}\text { Percent } \\
99 \\
99 \\
98 \\
97 \\
96\end{array}$ \\
\hline
\end{tabular}

tainable is seriously limited, especially at the low saturator temperatures. Fortunately, in electric hygrometer calibration and research, high air speeds are used in order to simulate radiosonde flights, hence relative humidities sufficiently high for all practical purposes can be obtained.

For any test chamber temperature, the lowest attainable relative humidity is limited by the lowest attainable saturator temperature, which is about $-55^{\circ} \mathrm{C}$. It is possible to reduce the relative humidity to about 16 percent at a test-chamber temperature of $-40^{\circ} \mathrm{C}$, and to lower values at higher test-chamber temperatures.

The saturator temperature must not be permitted to exceed room temperature, for then there is a possibility for the system above the saturator to assume a temperature less than that of the saturator with resultant condensation of water vapor. Equation 2 then would no longer give the relative humidity in the apparatus.

The internal diameter of the test chamber is 1 in. Two standard radiosonde electric hygrometers (4 in. $\times 5 / 16$ in. $\times 1 / 32$ in.) can be inserted into the test chamber. If all four channels or systems of the apparatus are operated simultaneously, then eight elements can be tested at one time.

The ratio of the absolute pressures in the test chamber and saturator, $P_{\mathrm{t}} / P_{\mathrm{s}}$, used in eq 2 , varies somewhat with air velocity and negligibly with temperature. Its variation with velocity may be once determined and then applied as a pressure correction. This correction is very small, and except for the high air velocities may be neglected for most work. Even for an air velocity of $1,000 \mathrm{ft} / \mathrm{min}$, the correction is only of the order of 0.6 percent. The pressure ratio corrections are shown in table 4.

TABLe 4. Pressure ratio corrections

\begin{tabular}{|r|c|}
\hline Air speed & $\begin{array}{c}\text { Pressure ratio } \\
\text { corrections, } \\
P_{t} / P_{s}\end{array}$ \\
\hline ft/min & \\
0 & 1.0000 \\
100 & 1.0000 \\
200 & 0.9997 \\
400 & .9989 \\
600 & .9978 \\
800 & .9962 \\
1,000 & .9938 \\
\hline
\end{tabular}

\section{Discussion and Summary}

An apparatus particularly suited for research and calibration of electric hygrometers of the Dunmore type has been designed, developed, and constructed. It operates by saturating air at one temperature and then raising the air temperature sufficiently to give any desired relative humidity. The important feature in this method is the continuous recirculation of air in a closed system and the use of a simple and efficient centrifugal saturator that can be used below $0^{\circ} \mathrm{C}$, as well as above. Air speeds up to 1,500 $\mathrm{ft} / \mathrm{min}$ are obtainable. A novel switching mechanism is utilized for giving rapid and discrete changes 
in relative humidity. This switching mechanism also is adaptable for giving rapid and discrete changes in temperature.

It is designed to operate at test-chamber temperatures above $0^{\circ} \mathrm{C}$, but may readily be used at temperatures as low as $-40^{\circ} \mathrm{C}$. The low temperatures are achieved, however, at some sacrifice of the maximum attainable relative humidity. For most of the test-chamber temperature range, practically any desired relative humidity may be produced by the apparatus.

Independent checks on the performance of the ap- paratus by means of the gravimetric method of moisture determination and the psychrometric method show an average agreement in relative humidity of 1.2 percent as measured and as given by the apparatus, eq 2.

The author gratefully acknowledges the assistance of R. A. Baum of the Shop Division in the construction of the apparatus and of E. T. Woolard in the experimental work.

Washington, August 14, 1950.

\title{
An Improved Apparatus for Determining Moisture in Rubber by Distillation with Toluene
}

\author{
By Max Tryon
}

\begin{abstract}
An improved apparatus has been developed for the determination of water in rubber by distillation with toluene. The volume of the water collected is measured in a graduated capillary tube, which allows more precise measurement than the tapered tube formerly used. The interior of the trap and condenser is coated with a water-repellent silicone polymer to prevent water droplets from adhering to the walls and to improve the accuracy. This paper describes the construction of the apparatus and a procedure for coating its interior with the silicone polymer. A method for calibration of the trap is included in a general procedure for use of the equipment.
\end{abstract}

\section{Introduction}

The water content of certain organic materials can be determined by a method that involves the formation of an azeotrope of an immiscible organic liquid with the water, distillation of the azeotrope, and separation of the water as a separate phase. The volume of water is then measured by the use of a graduated container.

For this purpose there are several devices commercially available for the collection and separation of water distilled with a liquid of low density, such as toluene. Since the time of Dean and Stark [1] ${ }^{1}$ there have been a large number of refinements and special applications similar to their original apparatus [2 to 9]. These refinements are essentially attempts to overcome the following shortcomings of the original design. First, there is a tendency for water to adhere to parts of the apparatus rather than to collect in the graduated portion. Second, there is a lack of precision of reading the volume of water collected due to the dimensions and shape of the graduated container. Third, sharp separation of the immiscible liquid and water does not always occur in apparatus with narrow water-collecting tubes, because the immiscible liquid tends to be trapped in the tube by the water. Fourth, the apparatus must be meticulously clean or the water will entrap the immiscible liquid and will not properly fill the measuring tube, regardless of the shape or dimensions of the tube.

\footnotetext{
1 Figures in brackets indicate the literature references at the end of this paper.
}

Subsequent modifications and refinements of the original apparatus have overcome some of these objections to varying degrees. The apparatus described in this paper, however, answers all these objections satisfactorily and also offers a few more advantages.

The procedure and apparatus described in this paper was originally devised in 1947 to determine small amounts of water (about $1 \%$ by weight) present in certain types of synthetic rubber $[10,11]$ that could not be analysed with procedures standard at that time. As a result, this procedure has been accepted as a referee method for determining moisture in all types of synthetic rubbers by the Subcommittee on Test Methods, Reconstruction Finance Corp., Office of Rubber Reserve.

It is felt that this apparatus supplies the need for a rapid, simple, precise method for determining small amounts of water in materials on a routine production basis and furnishes a valuable tool for research.

\section{Description of Apparatus}

The apparatus is shown schematically in figure 1. The distilling flask, $A$, contains the sample and toluene. This flask is connected to the collecting trap, $T$, by a $24 / 40$ standard taper joint lubricated with silicone stopcock grease. A West condenser equipped with a drip tip is connected to the trap by means of a 24/40 standard taper joint, also lubricated with silicone grease. The tapered portion of the trap 